Revista de

Contabilidade e

Organizações

www.rco.usp.br
DOI: http://dx.doi.org/10.11606/rco.v11i31.134473
Journal of

Accounting and

Organizations

\title{
Honorários de auditoria e Book-Tax Differences
}

Audit fees and Book-Tax Differences

Jéssica Rayse de Melo Silva Ávila ${ }^{a}$; Patrícia de Souza Costa ${ }^{a}$; Luiz Paulo Lopes Fávero ${ }^{\mathrm{b}}$

${ }^{a}$ Universidade Federal de Uberlândia

${ }^{b}$ Universidade de São Paulo

Palavras-chave

Honorários de auditoria.

Book-Tax Differences.

Riscos de auditoria.
Keywords

Audit fees.

Book-Tax Differences.

Audit risks.
Informações do Artigo

Recebido: 15 de julho de 2017

Aceito: 10 de janeiro de 2018

\section{Resumo}

As diferenças entre olucro contábile olucro tributável, denominadas internacionalmente de Book-Tax Differences (BTD), podem aumentar os riscos e esforços do auditor, elevando os honorários da auditoria. A pesquisa verifica se existe relação entre os tipos de BTD e os honorários de auditoria que foram pagos pelas companhias abertas brasileiras no período de 2010 a 2014. Diferente dos estudos internacionais, apenas a BTD positiva (lucro contábil maior que o lucro tributável) apresentou relação com os honorários. O maior gerenciamento de resultados e evasão fiscal, que geram a BTD positiva, historicamente presentes em países civil law, podem justificar essa diferença de resultados em relação a outros países em que os efeitos foram testados. A BTD positiva pode representar uma "bandeira vermelha" para auditores, investidores, analistas e reguladores avaliarem a qualidade das informações contábeis. Discute-se a relevância de aprofundar a análise de como os honorários de auditoria variam com os diversos tipos de BTD, em diferentes setores de atividade e origens legais de países.

\begin{abstract}
Differences between book and taxable income, internationally denominated BookTax Differences (BTD), can affect auditor's risks and efforts, raising audit fees. Thus, this article aims research is to verify if there is a relationship between the BTD types and the audit fees that were paid by the Brazilian public companies in the period from 2010 to 2014. Different from the international studies, only positive BTD (book income greater than the taxable income) was related to audit fees. The higher level of earnings management and tax evasion, which generate positive BTD, historically present in civil law countries may justify this difference in results regarding other observed countries. Positive BTD can represent a "red flag" for auditors, investors, analysts and regulators to assess the quality of accounting information. The article argues the relevance of enhance the analysis of audit fees and types of BTD, in different economic activities and countries'legal origin.
\end{abstract}

\section{Implicações práticas}

A diferença entre o lucro contábil e o lucro tributável aumenta o risco de perdas financeiras e de reputação da auditoria, e seus honorários. A diferença entre estes dois lucros, conhecida como Book-Tax Differences (BTD), é uma 'bandeira vermelha' para analistas, gestores e governo, de possível gerenciamento de resultados e evasão fiscal.

Copyright $($ C 2017 FEA-RP/USP. Todos os direitos reservados

\section{INTRODUÇ̃̃o}

A formação de preço do trabalho de auditoria deve contemplar os riscos assumidos pelos auditores e a possibilidade de ocorrência de perdas para esses profissionais (Gul, Chen \& Tsui, 2003; Oliveira, Souza \& Vieira, 2004). Hanlon, Krishnan e Mills (2012) sugerem que a Book-Tax Differences (BTD), diferença entre lucro contábil $(L C)$ e lucro tributável $(L T)$, pode ser um dos fatores capazes de criar complexidade adicional ao trabalho do auditor e aumentar os riscos advindos de problemas na qualidade da informação contábil, influenciando na determinação dos honorários de auditoria.

Autor Correspondente: Tel. (34) 3239-4411

E-mail: jessicarayse@hotmail.com (J. R. M. S. Ávila); patricia.costa@ufu.br (P. S. Costa); lpfavero@usp.br (L. P. L. Fávero)

Universidade Federal de Uberlândia. Av. João Naves de Ávila, 2121 - Santa Mônica, Uberlândia - MG, 38408-100, Brasil 
O subjetivismo responsável e as escolhas contábeis inerentes à adoção das International Financial Reporting Standards (IFRS) podem estar relacionados com o gerenciamento de resultado contábil e o fiscal, o que pode afetar os valores da BTD (Barth, Landsman \& Lang, 2008, Hanlon et al., 2012). O comportamento oportunístico degrada a qualidade da informação contábil (Iudícibus, Martins \& Carvalho, 2005) e aumenta o risco e a complexidade percebidos pelo auditor, que tenderá a refletir esses aspectos na precificação da prestação de seus serviços (Heltzer \& Shelton, 2011). Nesse contexto, Hanlon et al. (2012) verificaram que valores extremos de BTD podem indicar discricionariedade do gestor para maximização do lucro, o que influencia o risco percebido pelo auditor acerca da qualidade do lucro e da determinação dos honorários.

A possibilidade de a BTD ser oriunda de escolhas contábeis discricionárias dos gestores e, de algum modo, afetar a qualidade da informação, pode fazer com que os auditores considerem maiores esforços, riscos e complexidade na execução das atividades, afetando o planejamento dos trabalhos a serem realizados (Hribar, Kravet \& Wilson, 2014). Os impactos no planejamento podem ser traduzidos pela dedicação de mais horas de trabalho (ou elevação do custo da hora), pela contratação de profissionais mais especializados e pela aplicação de testes de auditoria mais robustos (Bedard \& Johnstone, 2004), fatores esses que podem onerar a precificação desse serviço.

Diante desse contexto, o artigo discute a relação entre os tipos de BTD e os honorários de auditoria pagos pelas companhias abertas brasileiras, portanto, em um país de origem civil law. Especificamente, o objetivo do estudo é verificar se os diversos tipos de $B T D$ afetam os honorários de auditoria que foram pagos pelas companhias abertas brasileiras. A amostra deste estudo é constituída por 246 companhias abertas brasileiras que negociaram ações na Bolsa de Mercadorias \& Futuros Bovespa (BM\&FBovespa) de 2010 a 2014.

Hanlon et al. (2012) ao analisarem companhias abertas dos Estados Unidos, país de origem common law, verificaram que a BTD total, a permanente e a temporária estão associadas à avaliação do risco e do esforço do auditor; e são capazes de elevar os honorários de auditoria. Martinez \& Lessa (2014) identificaram que a BTD total está associada com os honorários para companhias abertas brasileiras. Esta pesquisa se diferencia de Hanlon et al. (2012) e Martinez \& Lessa (2014), pois analisa a questão em um país de origem civil law como o Brasil, e testa ainda a BTD por seus tipos temporária, permanente, normal, anormal, positiva e negativa.

O histórico de maior vinculação entre normas fiscais e societária e menor BTD no caso brasileiro pode ter mudado após a adoção das IFRS (Iudícibus et al., 2005, Costa \& Lopes, 2015). Contudo, Jeanjean e Stolowy (2008) asseveram que a adoção das IFRS nos países de origem civil law impulsionou a prática do gerenciamento de resultados. Klann e Beuren (2011) identificaram que houve redução nos níveis de gerenciamento de resultados em empresas inglesas com a adoção das IFRS e aumento nas empresas brasileiras após essa adoção. Portanto, a expectativa é de que a adoção das IFRS em países civil law, como o Brasil, tenha influenciado a percepção dos auditores sobre a BTD nas informações contábeis e que estes equiponderaram seus riscos na sua remuneração.

Indo além de Hanlon et al. (2012), a análise nesta pesquisa inclui a BTD normal, anormal, positiva e negativa; o que pode trazer maior robustez aos resultados. Hanlon et al. (2012) conjecturam que os valores extremos de $B T D$ podem estar relacionados à um maior risco e aos honorários de auditoria em decorrência do gerenciamento de resultados. Contudo, tais autores não testaram a relação entre os honorários de auditoria e a $B T D$ anormal e a BTD positiva, as quais são derivadas do gerenciamento de resultados contábil e fiscal. Esta pesquisa avança testando essas relações.

Considerando-se que ainda não é concreto se há relação da BTD com os honorários de auditoria nos países civil law, este estudo procura contribuir para a construção do arcabouço teórico que apresenta a BTD como proxy para risco, complexidade e esforço do auditor, fatores esses que influenciam a precificação do trabalho da auditoria. Os resultados desta pesquisa podem ser úteis para auditores avaliarem como a BTD pode auxiliar na determinação dos honorários de auditoria, como também para analistas avaliarem a utilidade da $B T D$ na avaliação de riscos.

\section{REVISÃO DE LITERATURA}

\subsection{Relação entre tipos de btd e honorários de auditoria}

A BTD pode ser dividida conforme sua origem em normal e anormal; sua natureza em permanente e temporária e sua magnitude em positiva e negativa. Analisar a $B T D$ segregada por tipos é relevante, uma vez que alguns desses tipos podem estar mais relacionados a fatores que reduzem a qualidade da informação contábil como, por exemplo, ao conservadorismo e a persistência de resultados. 
A $B T D$ pode surgir especificamente da diferença entre normas contábeis e fiscais, resultando na $B T D$ normal, e de manipulações discricionárias sobre o lucro contábil $(L C)$ e o lucro tributável ( $L T)$, gerando assim a BTD anormal (Tang, 2005a, 2006).

A limitação dos critérios de mensuração dos resultados tributários, bem como a divergência de objetivo frente à norma societária, pode fazer com que, de uma mesma operação de negócios, sejam extraídos resultados diferentes (Mills, Newberry, \& Trautman, 2002). Isso acontece em razão de as IFRS permitirem uma diversidade maior de escolhas contábeis, consentindo aos gestores a opção de utilizar os métodos que mais se adequam à realidade econômico-financeira da empresa, o que, por vezes, diverge dos interesses do Fisco (Costa \& Lopes, 2015).

Por outro lado, a BTD anormal decorre do comportamento oportunístico dos gestores, configurado pelo gerenciamento de resultados $(G R)$ e pelo gerenciamento de tributos $(G T)$ (Tang, 2005b). Tang (2005b) defende ainda que o $G R$ e o $G T$ podem ocorrer simultaneamente, onde o gestor manipula tanto o $L C$ quanto o $L T$, não mantendo nenhum deles constante. Dessa forma, a análise da BTD anormal é dificultada, principalmente, pela falta de uma medida apropriada para mensurar o $G R$ e o $G T$ e pela confidencialidade das informações tributárias (Formigoni, Antunes \& Paulo, 2009). Esses fatores aumentam a complexidade para o auditor em determinar a origem da BTD anormal, demandado um esforço adicional e incrementando os riscos envolvidos, o que pode elevar os honorários requeridos (Hanlon et al., 2012).

A BTD permanente é aquela que jamais será equalizada entre as normas societária e fiscal por proceder do reconhecimento de valores que são registrados apenas sob tutela de uma das normas (Tang, 2005a, 2006). Encontram-se evidências de que a BTD permanente está fortemente associada com incertezas dos participantes do mercado sobre os resultados futuros das empresas, o que é um índício de que são potenciais fatores de risco para as companhias (Comprix, Graham Jr., \& Moore, 2011). Hanlon et al. (2012) comentam que a BTD permanente normalmente não é apontada como proxy para qualidade da informação contábil, pois não está relacionada ao reconhecimento de accruals, que é um aspecto inerente ao processo contábil. Entretanto, existem indícios de que altos valores de BTD permanente relacionam-se a uma menor persistência nos resultados, o que é um indicativo de degradação da qualidade do lucro (Marques, Costa, \& Silva, 2016). Ainda, estratégias bem elaboradas de minimização da carga tributária podem envolver as provisões do imposto de renda, uma das fontes da $B T D$ permanente, e resultar no desvio de recursos financeiros oriundos da prática de evasão fiscal (Desai \& Dharmapala, 2006). Desse modo, para verificar quanto a BTD permanente está afetando a qualidade da informação, o auditor deverá incorrer em maior esforço e correr mais riscos, o que pode resultar em uma associação positiva com os honorários requeridos.

A BTD temporária origina-se de transações que são reconhecidas em ambos os sistemas, mas que divergem quanto ao período da sua mensuração, podendo ocorrer "quando determinadas operações são reconhecidas contabilisticamente, mas não têm qualquer efeito em termos fiscais, ou vice-versa”" (Costa \& Lopes, 2015). Assim, possui caráter tempestivo, pois tem como característica a sua reversão em um dado momento (Hanlon et al., 2012). Comparada à BTD permanente, a parcela temporária da diferença entre o $L C$ e o $L T$ está menos associada às incertezas dos participantes de mercado, mas ainda assim, representa algum grau de risco sob a perspectiva dos resultados futuros das empresas (Comprix et al., 2011). Mas, assim como a BTD permanente, a parcela temporária está relacionada a menor persistência dos resultados, característica essa que indica menor qualidade dos lucros. Por essas razões, pode-se considerar que BTD temporária incrementa o risco para o auditor e afeta os honorários de auditoria (Hanlon et al., 2012).

A BTD positiva ocorre quando o $L C$ é maior que o $L T$, e pode envolver tanto as escolhas contábeis como o gerenciamento de resultados, com o objetivo de maximização do $L C$ e/ou a redução da carga tributária (Tang, 2005a, 2006). Em decorrência da necessidade de maiores esforços e a complexidade envolvida para identificar se a origem da $B T D$ é das escolhas contábeis inerentes à adoção das IFRS ou da manipulação discricionária do gestor, os auditores elevam os honorários diante da BTD positiva (Hanlon et al., 2012). Heltzer (2009) identificou que, nos anos em que as empresas apresentaram variações positivas nas $B T D$, exibiram aumento no reconhecimento tempestivo dos ganhos, ou seja, redução do conservadorismo condicional, o que pode ser um sinal de gerenciamento de resultados (Hanlon, 2005). Assim, a redução do conservadorismo diante da BTD positiva pode representar uma "bandeira vermelha" para o auditor, elevando a remuneração requerida por ele.

O fato do $L T$ ser maior do que o $L C$ na BTD negativa pode não incrementar os riscos de auditoria, porque reduz a chance de litígios com a autoridade fiscal do país, uma vez que o $L T$ maior resulta uma carga tributária mais elevada, e não é capaz de capturar a superestimação das receitas registradas contabilmente em relação às receitas fiscais (Hanlon et al., 2012). 
Por outro lado, Heltzer (2009) identificou que, nos anos em que as empresas apresentaram BTD negativa, essas exibiram maior conservadorismo incondicional no $L C$ e menor conservadorismo condicional e incondicional no $L T$. Tal fato aponta degradação na qualidade dos resultados, uma vez que o conservadorismo incondicional está relacionado às práticas de gerenciamento de resultados (Qiang, 2007). Logo, assim como os demais tipos de $B T D$, a BTD negativa pode relacionar-se positivamente com os honorários de auditoria por incrementar o risco do auditor.

Em resumo, Hanlon et al. (2012) encontraram relação positiva entre os honorários e a BTD total, a permanente e a temporária para companhias norte-americanas. Para o contexto brasileiro, Martinez \& Lessa (2014) testaram e encontraram a relação dos honorários apenas com a BTD total. Assim, a presente pesquisa avança a discussão analisando os tipos de $B T D$ analisados pelos citados autores, e adiciona a análise da $B T D$ normal, anormal, positiva e negativa, para o contexto brasileiro, como um caso de um país civil law. Portanto, coloca como hipótese de pesquisa: Os tipos de BTD estão positivamente relacionados com os honorários de auditoria.

Espera-se que o efeito da $B T D$ nos honorários de auditoria seja diferente dependendo se esta é positiva ou negativa. A literatura aponta que o fato de uma empresa apresentar $L C$ maior do que o $L T$ (BTD positiva) pode ser um indício de gerenciamento de resultados (Hanlon et al., 2012). Assim, espera-se que a BTD positiva tenha uma relação mais forte com os honorários de auditoria do que a BTD negativa.

\subsection{Outros determinantes dos honorários de auditoria}

O tamanho da empresa pode ser um fator de interferência na precificação dos honorários, já que grandes companhias possuem operações mais complexas, que exigem maior esforço e mais horas do profissional de auditoria (Simunic, 1980; Francis, 1984; Hallak \& Silva, 2012; Castro, Peleias \& Silva, 2015; Borges, Nardi \& Silva, 2017).

A quantidade de segmentos operacionais é apontada como elemento que aumenta substancialmente a complexidade das operações das empresas e impacta positivamente na determinação dos honorários de auditoria, porque aumenta a diversificação de relatórios financeiros da entidade e o número de centros de decisão a serem monitorados (Simunic, 1980).

Nessa linha de pensamento, as negociações no mercado externo como, por exemplo, a emissão de ADR's, apresentam maior complexidade no trabalho de auditoria, em virtude dos critérios que devem ser cumpridos em atendimento às bolsas de valores internacionais (Hanlon et al., 2012), o que pode elevar os honorários de auditoria.

Os honorários de auditoria podem, também, ser influenciados pela representatividade dos investimentos em estoques (Simunic, 1980, Borges et al., 2017) e pelos montantes expressivos de recebíveis (Wu, 2012). Auditar esses ativos demanda esforço e implica riscos para o auditor, que deve validar os métodos de mensuração e os critérios aplicados por meio de procedimentos de auditoria específicos que são recomendados para essas contas (Simunic, 1980; Wu, 2012).

Os accruals discricionários aumentam o risco de litígios para o auditor e elevam a necessidade de seu esforço (Hanlon et al., 2012). Embora a natureza dos accruals esteja relacionada à temporalidade do reconhecimento de valores no resultado, os gestores podem agir discricionariamente para aumentá-los ou reduzi-los com o intuito de gerenciar o resultado da entidade, o que pode deixar a associação do esforço e do risco do auditor mais evidente, elevando os honorários a serem cobrados (Martinez, 2008).

O status 'Big 4' da empresa de auditoria, também, pode influenciar o montante desses honorários requeridos. Essas empresas possuem mais recursos para o desenvolvimento do trabalho de auditoria, maior nível de especialização, maior competência e melhor reputação no trabalho de auditoria, por isso, cobram honorários mais caros por essa diferenciação na prestação de serviço (Francis, 1984; Yatim, Kent \& Clarkson, 2006; Hallak \& Silva, 2012; Castro et al., 2015; Borges et al., 2017).

Quando o trabalho de auditoria pode representar risco e perdas financeiras e morais, os honorários de auditoria refletem as diferenças entre os níveis de responsabilidade do auditor (Seetharaman, Gul \& Lynn, 2002). Para esses autores, os honorários de auditoria são maiores quando a firma de auditoria independente é propensa a emitir parecer com ressalvas.

As empresas que apresentam maior alavancagem financeira, maior grau de endividamento ou apresentam prejuízos, possuem maiores chances de insolvência, por isso aumentam os riscos para o profissional de auditoria, que, por sua vez, eleva os honorários (Arruñada, 1997, Castro et al., 2015). 
Por fim, empresas mais rentáveis oferecem menos riscos aos auditores, pois a possibilidade de essas apresentarem dificuldades financeiras é significativamente menor do que as não rentáveis, reduzindo os riscos e os honorários de auditoria (Hanlon et al., 2012; Borges et al.,2017).

\section{PROCEDIMENTOS METODOLÓGICOS}

A amostra desta pesquisa é constituída por companhias abertas brasileiras não financeiras que negociaram ações na BM\&FBovespa de 2010 a 2014. Foram excluídas as companhias pertencentes aos setores de "Fundos", "Seguros" e "Finanças", tendo em vista que esses possuem especificidades não aplicáveis aos demais setores. Também foram excluídas 101 companhias que não divulgaram os honorários de auditoria no período de estudo, resultando em uma amostra final disposta em um painel balanceado com 246 companhias.

Detalhes da amostra são apresentados no Material Suplementar anexo à esta pesquisa. O período 20102014 é pós-adoção das IFRS no Brasil e coincide com a emissão da Instrução Normativa nº 480/2009 da CVM, que tornou obrigatória a divulgação da remuneração do auditor no exercício.

Para testar a relação entre os honorários de auditoria $(H A)$ e a $B T D$ foi utilizado o modelo de Hanlon et al. (2012), especificado na Equação 1. As variáveis de controle utilizadas são: os accruals (ACC), se a firma de auditoria é uma Big4, o ativo total da empresa $(A T)$, o número de segmentos em que a empresa opera $(S E G)$, a complexidade das operações pela emissão de ADR, os estoques (EST), os recebíveis (REC), o endividamento $(E N D)$, a rentabilidade pelo EBIT (REN), se foi apurado resultado negativo no período ( $N E G)$ e, por fim, a opinião da auditoria $(O P I N)$. Para maiores esclarecimentos sobre a descrição e o cálculo das variáveis utilizadas nas Equações 1 a 7, sugere-se a consulta ao já mencionado Material Suplementar.

$$
\begin{gathered}
H A_{i t}=\alpha_{i}+\beta_{1} B T D_{i t}+\beta_{2} A C C R_{i t}+\beta_{3} B I G 4_{i t}+\beta_{4} A T_{i t}+\beta_{5} S E G_{i t}+\beta_{6} A D R_{i t}+\beta_{7} E S T_{i t}+ \\
\beta_{8} R E C_{i t}+\beta_{9} D I V_{i t}+\beta_{10} R E N_{i t}+\beta_{11} N E G_{i t}+\beta_{12} O P I N_{i t}+\varepsilon_{i t}
\end{gathered}
$$

O modelo especificado na Equação 1 foi testado para cada tipo de BTD de forma separada: total, permanente, temporária, positiva, negativa, normal e anormal.

A BTD total é a diferença total entre o $L C$ e o $L T$ (Equação 2). Neste estudo, o $L C$ utilizado é o Lucro Antes do Imposto de Renda (LAIR), uma vez que esse é o lucro societário final da empresa antes da dedução dos impostos. O LT não é amplamente evidenciado pelas companhias brasileiras nas notas explicativas, por isso, essa variável é estimada pela extrapolação da Despesa de Imposto de Renda do período (DIRC) menos a Despesa com Imposto de Renda Diferido (DIRD), dividida pela alíquota máxima de Imposto de Renda (A).

$$
\mathrm{BTD}_{\text {total }} \mathrm{it}_{\mathrm{it}}=\log \left(\mathrm{IAIR}_{\mathrm{it}}-\left(\frac{\left(\mathrm{DIRC}_{\mathrm{it}}-\mathrm{DIRD}_{\mathrm{it}}\right)}{\mathrm{A}_{\mathrm{it}}}\right) \mathrm{l}\right)
$$

Para a estimação da BTD permanente e da BTD temporária, utilizou-se as Equações 3 e 4, respectivamente:

$$
\begin{aligned}
& \text { BTD permanente }_{i t}=\log \left(\left|\left(\frac{B T D \text { total }_{i t}-D I R D}{A_{i t}}\right)\right|\right) \\
& B T D \text { temporária } a_{i t}=\log \mid \text { DIRC। }
\end{aligned}
$$

A BTD positiva e a BTD negativa foram calculadas respectivamente conforme as Equações 5 e 6 :

$$
\begin{aligned}
& \text { BTD positiva }_{i t}=\log \left(\left|\mathrm{LAIR}_{i t}-\mathrm{LT}_{i t}\right|\right), \text { se } \mathrm{LC}_{i t}>\mathrm{LT}_{i t} \\
& \text { BTD negativa }_{i t}=\log \left(\left|\mathrm{LAIR}_{i t}-\mathrm{LT}_{i t}\right|\right), \text { se } \mathrm{LC}_{i t}<\mathrm{LT}_{i t}
\end{aligned}
$$


Para estimação das parcelas normal e anormal da BTD total utilizou-se a Equação 7, que é uma adaptação do modelo de Tang (2006) e Martinez e Passamani (2014):

$$
\text { BTD total }_{\text {it }}=\alpha_{i}+\beta_{1} \mathrm{INV}_{\mathrm{it}}+\beta_{2} \Delta \mathrm{REV}_{\mathrm{it}}+\beta_{3} \mathrm{NOL}_{\mathrm{it}}+\beta_{4} \mathrm{DIRD}_{\mathrm{it}}+\beta_{5}(\mathrm{PL}-\mathrm{LL})_{\mathrm{it}}+\beta_{6} \Delta \mathrm{EST}_{\mathrm{it}}+\varepsilon_{i t}
$$

A BTD anormal foi obtida pela estimação dos resíduos (cit) da Equação 7 e a BTD normal foi calculada subtraindo-se a BTD anormal da BTD total.

\section{RESULTADOS E DISCUSSÕES}

\subsection{Análise descritiva}

O valor dos honorários de auditoria equivale a aproximadamente $\mathrm{R} \$ 169$ mil (5,227 - valor na tabela $1 \mathrm{em}$ ordem logarítmica). Verifica-se que $25 \%$ das companhias pagam honorários anuais de R\$ 53 mil $(4,724)$ e mais de $50 \%$ remunera a auditoria acima de $\mathrm{R} \$ 230$ mil.

Tabela 1. Estatística descritiva

\begin{tabular}{lrrrrrrrr}
\hline Variável & $\mathbf{n}$ & Média & $\begin{array}{c}\text { Desvio } \\
\text { padrão }\end{array}$ & Mínimo & $\mathbf{2 5 \%}$ & Mediana & $\mathbf{7 5 \%}$ & Máximo \\
\hline Honorários de auditoria & 1.230 & 5,227 & 0,732 & 3,466 & 4,724 & 5,362 & 5,708 & 7,002 \\
BTD total & 1.132 & 4,854 & 1,247 & 1,869 & 4,211 & 5,096 & 5,771 & 7,000 \\
BTD temporária & 846 & 4,030 & 0,888 & 1,602 & 3,476 & 4,107 & 4,605 & 5,977 \\
BTD permanente & 1132 & 4,896 & 1,247 & 1,869 & 4,260 & 5,167 & 5,803 & 7,015 \\
BTD normal & 826 & 5,186 & 0789 & 3,222 & 4,533 & 5,237 & 5,773 & 6,968 \\
BTD anormal & 826 & 0,000 & 0,335 & $-2,106$ & $-0,099$ & 0,031 & 0,150 & 1,083 \\
BTD positiva & 805 & 5,201 & 1,021 & 1,869 & 4,660 & 5,369 & 5,916 & 7,000 \\
BTD negativa & 327 & 4,000 & 1,341 & 1,869 & 2,674 & 4,228 & 5,031 & 6,928 \\
Accruals & 1.222 & 4,518 & 1,320 & 0,091 & 3,957 & 4,765 & 5,400 & 6,873 \\
Ativo & 1.227 & 5,843 & 1,415 & 1,000 & 5,375 & 6,176 & 6,704 & 8,332 \\
Segmentos & 1.230 & 0,108 & 0,213 & 0,000 & 0,000 & 0,000 & 0,000 & 0,778 \\
Estoques & 1.218 & 0,080 & 0,108 & 0,000 & 0,000 & 0,012 & 0,137 & 0,446 \\
Recebíveis & 1.218 & 0,162 & 0,179 & 0,000 & 0,033 & 0,111 & 0,220 & 0,887 \\
Endividamento & 1.222 & 0,279 & 0,258 & 0,000 & 0,049 & 0,261 & 0,428 & 1,405 \\
Rentabilidade & 1.222 & $-0,256$ & 1,823 & $-15,200$ & $-0,008$ & 0,054 & 0,116 & 0,470 \\
\hline
\end{tabular}

Fonte: Elaborada pelos autores.

Nota-se que, com exceção da BTD anormal, para todos os tipos de BTD, a média superou 4,00 (Tabela 1). Esses resultados diferenciam-se do apresentado por Hanlon et al. (2012), que encontraram valores próximos de 2,00. Esses achados sugerem que as $B T D$ das companhias abertas brasileiras são maiores quando comparada àquelas das empresas norte-americanas, corroborando com André, Broye, Pong e Schatt (2011), que apontaram sobre a tendência de a BTD ser maior em países de origem civil law. Para as variáveis de controle, as médias se assemelharam às encontradas por Hanlon et al. (2012), exceto para os accruals que apresentaram um desalinhamento com o resultado internacional $(4,518$ contra 2,634$)$.

Observa-se que os honorários de auditoria apresentaram associação positiva e significativa com todas as variáveis de controle (Tabela 2). Todavia, para a rentabilidade, o sinal diferenciou-se do esperado, sugerindo que quanto maior a rentabilidade da companhia maior será o honorário de auditoria. 
Tabela 2. Matriz de correlação entre as variáveis do modelo e os tipos de BTD

\begin{tabular}{llccccccc}
\hline & $\begin{array}{c}\text { Honor. } \\
\text { Auditoria }\end{array}$ & $\begin{array}{c}\text { BTD } \\
\text { total }\end{array}$ & $\begin{array}{c}\text { BTD } \\
\text { tempor. }\end{array}$ & $\begin{array}{c}\text { BTD } \\
\text { perman. }\end{array}$ & $\begin{array}{c}\text { BTD } \\
\text { anormal }\end{array}$ & $\begin{array}{c}\text { BTD } \\
\text { normal }\end{array}$ & $\begin{array}{c}\text { BTD } \\
\text { positiva }\end{array}$ & $\begin{array}{c}\text { BTD } \\
\text { negativa }\end{array}$ \\
\hline Honor. Auditoria & 1,000 & $0,581^{* *}$ & $0,220^{* *}$ & $0,595^{* *}$ & $-0,0292$ & $0,352^{* *}$ & $0,435^{* *}$ & $0,690^{* *}$ \\
Accruals & $0,563^{* *}$ & $0,839^{* *}$ & $0,582^{* *}$ & $0,847^{* *}$ & $0,159^{* *}$ & $0,681^{* *}$ & $0,754^{* *}$ & $0,904^{* *}$ \\
Ativo & $0,614^{* *}$ & $0,876^{* *}$ & $0,713^{* *}$ & $0,882^{* *}$ & $0,102 * *$ & $0,857^{* *}$ & $0,860^{* *}$ & $0,875^{* *}$ \\
Segmentos & $0,147^{* *}$ & $0,242^{* *}$ & $0,142^{* *}$ & $0,241^{* *}$ & 0,0188 & $0,183^{* *}$ & $0,191^{* *}$ & $0,332^{* *}$ \\
Estoques & $0,155^{* *}$ & $0,109^{* *}$ & $-0,170^{* *}$ & $0,099^{* *}$ & $-0,035$ & $-0,160^{* *}$ & $-0,039$ & $0,259^{* *}$ \\
Recebíveis & $0,114^{* *}$ & $0,142^{* *}$ & $-0,096^{* *}$ & $0,145^{* *}$ & $-0,009$ & $-0,115^{* *}$ & $-0,004$ & $0,184^{* *}$ \\
Endividamento & $0,256^{* *}$ & $0,322^{* *}$ & $0,127^{* *}$ & $0,328^{* *}$ & 0,034 & $0,079^{* *}$ & $0,232^{* *}$ & $0,434^{* *}$ \\
Rentabilidade & $0,286^{* *}$ & $0,370^{* *}$ & $-0,031$ & $0,376^{* *}$ & 0,043 & $0,200^{* *}$ & $0,361^{* *}$ & $0,406^{* *}$ \\
\hline
\end{tabular}

Fonte: Elaborada pelos autores.

Nota:**denota a significância estatística a 5\%.

O valor do coeficiente de correlação de 0,581 , identificado para a BTD total, na Tabela 4, está em harmonia com aquele encontrado por Hanlon et al. (2012) de 0,528 para as companhias norte-americanas. Assim, embora o coeficiente de correlação não permita determinar causalidade nessa relação, pode-se dizer que o comportamento da $B T D$ auxilia a compreensão do comportamento dos honorários de auditoria e vice-versa. Os demais tipos de $B T D$, exceto a $B T D$ anormal, estão positivamente relacionados com os honorários, o que sugere que as companhias que apresentam maior $B T D$ representam maior risco e complexidade, o que pode elevar os honorários dos auditores.

\subsection{Análise multivariada}

Na Tabela 3, são apresentados os resultados dos testes da Equação 1 para todos os tipos de BTD, sendo as equações para BTD temporária e BTD positiva estimadas por efeitos aleatórios e as equações para os demais tipos de BTD estimadas por efeitos fixos, conforme resultados dos testes Chow, Breusch e Pagan e Hausman.

A BTD positiva está relacionada com os honorários de auditoria (Tabela 3). Especificamente, para cada $1 \%$ de aumento na BTD positiva, há um aumento de $8,8 \%$ nos honorários (coeficiente $\beta_{1}$ ). O $L C$ superior ao $L T$ pode representar maior risco de perda e maior esforço dos auditores, o que eleva a remuneração desses profissionais. $\mathrm{O}$ fato da BTD positiva poder incluir, tanto o gerenciamento de resultados quanto as práticas de evasão fiscal, podem justificar a relação entre essa variável e os honorários de auditoria (Hanlon et al., 2012).

Outra possível explicação é o fato da BTD positiva estar relacionada negativamente com o conservadorismo condicional (Heltzer, 2009). Isso sugere que as empresas, as quais apresentam BTD positiva, reconhecem de maneira mais tempestiva os ganhos, o que pode ser um sinal de gerenciamento de resultados. Infere-se que o auditor se sente exposto a maiores riscos com a redução do conservadorismo condicional, elevando seus honorários.

Os demais tipos de BTD não apresentaram relação com a remuneração dos auditores (Tabela 3). Esses resultados diferenciam-se daqueles encontrados por Hanlon et al. (2012) e Martinez \& Lessa (2014). Uma possível justificativa para esse resultado pode ser a origem civil law do Brasil, e a forma como os ajustes fiscais são realizados sobre o $L C$. O volume de ajustes realizados pode desestimular os auditores à fazerem uma análise mais aprofundada da $B T D$, levando-os a interpretar que esses tipos de $B T D$ não incrementam o risco da auditoria, por isso não são refletidos nos honorários requeridos. Já em relação ao estudo de Martinez \& Lessa (2014), uma possível explicação para a diferença de resultados pode ser o período da amostra (2009 a 2011) e o estágio de adoção parcial das IFRS. A curva de aprendizagem na adoção das IFRS (Costa \& Lopes, 2015) pode justificar essa distinção de resultados.

O status da firma de auditoria influencia os honorários. Esse resultado corrobora aqueles de Simunic (1980), Francis (1984), Yatim et al. (2006) e Martinez \& Lessa (2014), que sugeriram que há incremento nesses honorários em virtude da elevada reputação e competência das Big4. 
Tabela 3. Impactos dos tipos de BTD nos Honorários de Auditoria (Equação 1)

\begin{tabular}{|c|c|c|c|c|c|c|c|c|}
\hline Variáveis & & $\begin{array}{l}\text { BTD } \\
\text { Total } \\
\end{array}$ & BTD permanente & BTD anormal & BTD normal & BTD negativa & BTD temporária & BTD positiva \\
\hline \multirow[t]{2}{*}{$\mathrm{BTD}^{1}$} & $\beta_{1}$ & 0,012 & 0,044 & $-0,018$ & 0,833 & $-0,038$ & $-0,002$ & 0,088 \\
\hline & Estat. & 0,420 & 1,180 & $-0,570$ & 1,840 & $-0,670$ & $-0,080$ & $2,160^{* *}$ \\
\hline \multirow[t]{2}{*}{ Accruals } & $\beta_{2}$ & $-0,026$ & $-0,031$ & $-0,018$ & $-0,020$ & 0,025 & $-0,018$ & $-0,043$ \\
\hline & Estat. & $-1,250$ & $-1,450$ & $-0,780$ & $-0,880$ & 0,750 & $-0,720$ & $-1,560$ \\
\hline \multirow[t]{2}{*}{ BIG4 } & $\beta_{3}$ & 0,155 & 0,152 & 0,146 & 0,141 & 0,171 & 0,170 & 0,233 \\
\hline & Estat. & $3,770^{* * *}$ & $3,680^{* * *}$ & $3,220 * * *$ & $2,910 * * *$ & $1,920^{*}$ & $4,210^{* * *}$ & $4,780 * * *$ \\
\hline \multirow[t]{2}{*}{ Ativo } & $\beta_{4}$ & 0,004 & $-0,003$ & 0,197 & 0,198 & 0,002 & 0,264 & 0,235 \\
\hline & Estat. & 0,090 & $-0,070$ & 1,650 & 1,620 & 0,050 & $4,030 * * *$ & $3,720 * * *$ \\
\hline \multirow[t]{2}{*}{ Segmentos } & $\beta_{5}$ & $-0,068$ & $-0,067$ & $-0,042$ & $-0,033$ & 0,161 & $-0,056$ & $-0,112$ \\
\hline & Estat. & $-0,680$ & $-0,670$ & $-0,370$ & $-0,300$ & 1,180 & $-0,530$ & $-0,990$ \\
\hline \multirow[t]{2}{*}{ Complexidade das Operações } & $\beta_{6}$ & 0,060 & 0,063 & 0,076 & 0,068 & 0,089 & 0,035 & $-0,050$ \\
\hline & Estat. & 0,810 & 0,840 & 0,770 & 0,700 & 0,690 & 0,390 & $-0,580$ \\
\hline \multirow[t]{2}{*}{ Estoques } & $\beta_{7}$ & $-0,135$ & $-0,118$ & $-0,130$ & $-0,095$ & $-0,426$ & 0,518 & 0,580 \\
\hline & Estat. & $-0,240$ & $-0,210$ & $-0,230$ & $-0,170$ & $-0,630$ & 1,490 & $1,950^{*}$ \\
\hline \multirow[t]{2}{*}{ Recebíveis } & $\beta_{8}$ & $-0,569$ & $-0,574$ & $-0,731$ & $-0,790$ & $-0,246$ & $-0,278$ & $-0,222$ \\
\hline & Estat. & $-3,040 * * *$ & $-3,060 * * *$ & $-2,650 * * *$ & $-2,730 * * *$ & $-1,870^{*}$ & $-1,290$ & $-1,240$ \\
\hline \multirow[t]{2}{*}{ Endividamento } & $\beta_{9}$ & 0,030 & 0,027 & 0,022 & 0,108 & 0,078 & $-0,091$ & 0,066 \\
\hline & Estat. & 0,420 & 0,380 & 0,210 & 0,920 & 1,510 & $-0,800$ & 0,510 \\
\hline \multirow[t]{2}{*}{ Rentabilidade } & $\beta_{10}$ & 0,017 & 0,018 & 0,050 & 0,120 & 0,021 & 0,019 & $-0,030$ \\
\hline & Estat. & 1,610 & $1,720^{*}$ & 0,410 & 0,880 & $1,800^{*}$ & 0,130 & $-0,140$ \\
\hline \multirow[t]{2}{*}{ Resultado Negativo } & $\beta_{11}$ & 0,055 & 0,054 & 0,043 & 0,120 & 0,086 & 0,041 & $-0,007$ \\
\hline & Estat. & 1,610 & 1,610 & 1,040 & 0,880 & $3,130 * * *$ & 0,900 & $-0,100$ \\
\hline \multirow[t]{2}{*}{ Opinião da auditoria } & $\beta_{12}$ & $-0,025$ & $-0,021$ & $-0,001$ & 0,011 & 0,003 & $-0,054$ & 0,038 \\
\hline & Estat. & $-0,440$ & $-0,370$ & $-0,020$ & 0,140 & 0,060 & $-0,690$ & 0,330 \\
\hline \multirow[t]{2}{*}{ Cons } & $\alpha$ & 5,269 & 5,174 & 4,276 & $-0,071$ & 4,892 & 3,750 & 3,453 \\
\hline & Estat. & $22,000 * * *$ & $21,740 * * *$ & $5,830 * * *$ & $-0,030$ & $20,290 * * *$ & $9,690 * * *$ & $13,220 * * *$ \\
\hline R-sq: within & & 0,029 & 0,031 & 0,029 & 0,038 & 0,107 & & \\
\hline Prob. $>$ F & & 0,000 & 0,000 & 0,003 & 0,001 & 0,000 & & \\
\hline R-sq: overall & & & & & & & 0,098 & 0,242 \\
\hline Prob.> chi2 & & & & & & & 0,000 & 0,000 \\
\hline
\end{tabular}

Nota: $* * * * * \mathrm{e} *$ denotam significância estatística a 1\%, 5\% e 10\%, respectivamente. 1 - representa os tipos de $B T D$; a Equação 1 foi testada sete vezes, sendo uma vez para cada tipo de $B T D$. 
O sinal negativo da variável Recebíveis é contrário àquele apontado por Simunic (1980) e Hanlon et al. (2012). Para todos os modelos, a estatística VIF para Recebíveis foi analisada, ficando abaixo de 2,0. Desse modo, a hipótese de que a multicolinearidade pudesse ter influenciado a inversão de sinal foi descartada em todos os casos.

Testes de sensibilidade foram realizados, substituindo a fórmula de cálculo da BTD especificada na Equação 2, por aquelas utilizadas por Costa e Lopes (2015) para o contexto brasileiro e os resultados foram semelhantes àqueles mostrados na Tabela 3. Outro teste realizado foi a aplicação da Equação 1 por setor de atividade. Nesse contexto, a BTD temporária apresentou relação com os honorários de auditoria para os setores de Alimentos e Bebidas, Máquinas Industriais, Química, Software e Dados e Veículos e Peças; enquanto a BTD permanente para o setor de Alimentos e Bebidas. A BTD negativa foi significante para os setores de Siderurgia e Metalurgia, Telecomunicações e Têxtil, e a BTD anormal para o setor de Veículos e Peças. Esses resultados sugerem que analisar a $B T D$ por setor é relevante para avaliar o impacto dessa variável nos honorários de auditoria.

\section{CONSIDERAÇÕES FINAIS}

Os honorários de auditoria estão associados apenas à BTD positiva. Esse tipo de BTD pode ser oriundo de operações de fusão, cisão e incorporação, planejamento tributário, aproveitamento de benefícios e prejuízos fiscais, conservadorismo condicional e manipulações discricionárias. Infere-se que, a verificação das causas para o surgimento da BTD positiva torna a avaliação de risco do gestor mais criteriosa, e representa uma "bandeira vermelha" para a possibilidade de processos movidos pela autoridade tributária pela prática de evasão fiscal, perdas financeiras e a perda da reputação da firma de auditoria. Esses riscos, somados ao esforço que o auditor demandará para determinar a origem da BTD positiva, implicam na elevação dos honorários de auditoria.

Diferente de Hanlon et al. (2012), para um país civil law o que mais afetou os honorários de auditoria foi a diferença positiva entre lucro contábil e o lucro tributável, o que provavelmente se deve ao histórico de maior nível de gerenciamento de resultados e evasão fiscal nesse tipo de país, inclusive impulsionado após a adoção das IFRS (ver Jeanjean \& Stolowy, 2008).

A redução do conservadorismo condicional na presença de maior volume de BTD positiva (Heltzer, 2009) pode, também, ser uma explicação para a elevação dos honorários de auditoria. Os auditores podem esperar maior exposição a riscos diante de um reconhecimento mais tempestivo de ganhos, requerendo maior remuneração para a realização de testes adicionais e mais robustos. Esse possível desconforto do auditor diante da BTD positiva, derivada da redução do conservadorismo condicional, pode ainda ser um resquício da cultura conservadora do contador no registro de perdas e ganhos.

Os resultados desta pesquisa contribuem com a literatura ao demonstrar a relevância de pesquisar a relação dos honorários de auditoria com a BTD segregada por tipos, por setor e por origem civil e common law.

Por fim, os resultados podem levar auditores, investidores, analistas, reguladores e gestores a considerar o papel da BTD positiva como uma "bandeira vermelha" quanto ao gerenciamento de resultados e a evasão fiscal.

\section{REFERÊNCIAS}

André, P., Broye, G., Pong, C. K. M, \& Schatt, A. (2011). Audit fees, big four premium and institutional settings: the devil is in the details! SSRN Working Paper.

Arruñada, B. (1997). La calidad de la auditoria: incentivos privados y regulacion. Madrid: Marcial Pons.

Barth, M. E., Landsman, W. R. \& Lang, M. H. (2008). International accounting standards and accounting quality. Journal of Accounting Research, 46(3), 467-498.

Bedard, J., \& Johnstone, K. (2004). Earnings manipulation risk, corporate governance risk, and auditor is planning and pricing decisions. The Accounting Review, 79, 277-304.

Borges, V., Nardi, P., \& Silva, R. (2017). Determinantes dos Honorários de Auditoria das Empresas Brasileiras de Capital Aberto. Contabilidade, Gestão e Governança, 20(2), 216-230. 
Castro, W., Peleias, I., \& Silva, G. (2015). Determinantes dos Honorários de Auditoria: um Estudo nas Empresas Listadas na BM\&FBOVESPA, Brasil.Revista Contabilidade \& Finanças, 26(69), 261-273.

Comprix, J., Graham Jr. R. C., \& Moore, J. A. (2011). Empirical evidence on the impact of book-tax differences on divergence of opinion among investors. Journal of the American Taxation Association, 33(1), 57-78.

Costa, P. S., \& Lopes, A. B. (2015). Implicações da adoção das IFRS sobre as book-tax differences: o caso do Brasil. Alemanha: NEA.

Desai, M. A., \& Dharmapala, D. (2006). Corporate tax avoidance and high-powered incentives. Journal of Financial Economics, 79(1), 145-179.

Formigoni, H., Antunes, M. T. P., \& Paulo, E. (2009). Diferença entre o Lucro Contábil e Lucro Tributável: uma análise sobre o gerenciamento de resultados contábeis e gerenciamento tributário nas companhias abertas brasileiras. Brazilian Business Review, 6, 44-61.

Francis, J. R. (1984). The effect of audit firm size on audit prices. Journal of Accounting and Economics, 6, 133151.

Gul, A., Chen, C. J. P, \& Tsui, J. S.L. (2003). Discretionary Accounting Accruals, Managers' Incentives, and Audit Fees. Contemporary Accounting Research, 20(3), 441-464.

Hallak, R. T. P., \& Silva, A. L. C. (2012). Determinantes das despesas com serviços de auditoria e consultoria prestados pelo auditor independente no Brasil. Revista Contabilidade \& Finanças, 23(60), 223-231.

Hanlon, M. (2005). The persistence and pricing of earnings, accruals, and cash flows when firms have large booktax differences. The accounting review, 80(1), 137-166.

Hanlon, M., Krishnan, G. V.,\& Mills, L. F. (2012). Audit Fees and Book-Tax Differences. The Accounting Review, 34(1), 55-86.

Heltzer, W, \& Shelton, S. W (2011). Book-tax differences and audit risk: evidence from the United States. Working paper, DePaul University.

Heltzer, W. (2009). Conservatism and book-tax differences. Journal of Accounting, Auditing and Finance, 24(3), 469-504.

Hribar, P., Kravet, T., \&Wilson, R. (2014). A new measure of accounting quality. Review of Accounting Studies, 19(1), 506-538.

Iudícibus, S., Martins, E., \& Carvalho, L. N. (2005). Contabilidade: aspectos relevantes da epopeia de sua evolução. Revista Contabilidade \& Finanças, 16(38), 7-19.

Jeanjean, T., \& Stolowy, H. (2008). Do accounting standards matter? An exploratory analysis of earnings management before and after IFRS adoption. Journal of Accounting Public Policy, 27, 480-494.

Klann, R. C., \& Beuren, I. M. (2011). Efeitos da convergência contábil às IFRS no gerenciamento de resultados de empresas europeias. Anais do Congresso ANPCONT, Vitória, ES, Brasil, 5.

Marques, A. V. C., Costa, P. S., \& Silva, P. R. (2016). Relevância do Conteúdo Informacional das Book-Tax Differences para Previsão de Resultados Futuros: Evidências de Países-Membros da América Latina. Revista Contabilidade \& Finanças, 27(70), 29-42.

Martinez A. L. (2008). Detectando Earnings Management no Brasil: Estimando os Accruals discricionários. Revista de Contabilidade \& Finanças, 19, 7-17.

Martinez, A. L., \& Passamani, R. R. (2014). Book-Tax Differences e sua Relevância Informacional No Mercado de Capitais No Brasil. Revista de Gestão, Finanças e Contabilidade, 4(2), 20-37. 
Martinez, A.L., \& Lessa, R. C. (2014). The Effect of Tax Aggressiveness and Corporate Governance on Audit Fees: Evidences from Brazil. Journal of Management Research, 6(1), 95-108.

Mills, L. F., Newberry, K., \& Trautman, W.B. (2002). Trends in book-tax income and balance sheet differences. SSRN. Recuperado de http://papers.ssrn.com/sol3/papers.cfm?abstract_id=313040.

Qiang, X. (2007). The effects of contracting, litigation, regulation, and tax costs on conditional and unconditional conservatism: Cross-sectional evidence at the firm level. The Accounting Review, 82(3), 759-796.

Oliveira, J. L. R., Souza, A. A., \& Vieira, C. M. (2004). Análise dos fatores envolvidos na estimação de custos e formação de preço das empresas de auditoria. Contabilidade Vista \& Revista, 15(2), 107-125.

Seetharaman, A., Gul, F. A., \& Lynn, S. G. (2002). Litigation risk and audit fees: Evidence from UK firms crosslisted on US markets. Journal of Accounting and Economics, 33(1), 91-115.

Simunic, D. (1980). The pricing of audit services: theory and evidence. Journal of Accounting Research, 18, 161190.

Tang, T. Y. H. (2005a). Book-Tax Differences, a Proxy for Earnings Management and Tax Management - Empirical Evidence from China. SSRN Electronic Journal, 1-36.

Tang, T. Y. H. (2005b). The Market Perception of BTD: an empirical study in China's capital markets. The Accounting Review.

Tang, T. Y. H. (2006). The Value Relevance of Book-Tax Differences - An Empirical Study in China's Capital Market. SSRN Eletronic Journal.

Wu, X. (2012). Corporate governance and audit fees: Evidence from companies listed on the Shanghai Stock Exchange. China Journal of Accounting Research, 5(4), 321-342.

Yatim, P., Kent, P., \& Clarkson, P. (2006). Governance structures, ethnicity, and audit fees of Malaysian listed firms. Managerial Auditing Journal, 21(7), 757-782. 


\section{MATERIAL SUPLEMENTAR}

A amostra desta pesquisa foi constituída por companhias abertas brasileiras que negociaram ações na BM\&FBovespa de 2010 a 2014. Esse período foi escolhido por contemplar a adoção das IFRS no Brasil, apoiando-se na perspectiva de que a abordagem baseada em princípios poderia estimular os gestores a efetuar mais escolhas contábeis (Agoglia, Doupnik \& Tsakumi, 2011), aumentando a BTD. Ainda, coincide com a emissão da Instrução Normativa da CVM n ${ }^{\circ} 480$, de 7 de dezembro de 2009, que instituiu a obrigatoriedade da divulgação de informações relativas aos auditores independentes, como a descrição dos serviços contratados e montante total de remuneração no último exercício social.

Os dados para a realização da pesquisa foram coletados na base Economática $^{\mathrm{TM}}$, e posteriormente conferidos com as demonstrações financeiras divulgadas pelas companhias na Comissão de Valores Mobiliários (CVM), considerando um critério de amostragem aleatória. Essa conferência foi realizada com o intuito de verificar inconsistências nas informações, como dados incorretos, inversões de sinais, entre outros. A Opinião de Auditoria foi obtida no site da CVM, assim como as Informações sobre Segmentos (Notas Explicativas) e os honorários de auditoria (Formulário de Referência).

Foram consideradas as companhias pertencentes aos 20 setores básicos do Economática ${ }^{\mathrm{TM}}$, com exceção daquelas pertencentes a "Fundos", "Seguros" e "Finanças", tendo em vista que esses possuem especificidades não aplicáveis aos demais setores. Foram eliminadas as observações que não continham informações divulgadas em relação à variável dependente. $\mathrm{O}$ critério implicou na exclusão completa (de todos os períodos) das companhias que omitiram os honorários de auditoria em um ou mais períodos. A Tabela 1 resume o tratamento da amostra.

Tabela 1. Tratamento dos dados

\begin{tabular}{rcc}
\hline & $\begin{array}{c}\text { Companhias } \\
\text { (por ano) }\end{array}$ & $\begin{array}{c}\text { Observações } \\
\text { (total da amostra) }\end{array}$ \\
\hline (-) Compervações iniciais & 347 & 1.735 \\
$(=)$ Amostra final & 246 & $(505)$ \\
\hline
\end{tabular}

Fonte: Elaborada pelos autores.

Existiam duas formas distintas de mitigar a influência dos outliers na amostra, sendo a primeira o processo de winsorização, "que elimina, de forma ordenada, um número igual de observações de cada lado da distribuição", e a segunda pela exclusão completa da observação, resultando na indesejada redução da amostra, do ponto de vista estatístico (Fávero, Belfiore, Silva, \& Chan, 2009, p.56). Considerando que a amostra já havia sido reduzida pela exclusão das observações com dados faltantes da variável dependente, optou-se pela a winsorização a $1 \%$.

Os tipos de BTD para as três especificações de dados em painel - pooled, efeitos fixos e efeitos aleatórios -, foram testadas no software Stata $12^{\circledR}$. Para a definição do modelo mais adequado para os dados, utilizaram-se os testes de Chow, Breusch e Pagan e Hausman.

Analogamente ao estudo de Hanlon et. al (2012), a principal variável independente desta pesquisa é a BTD. Para sua estimação, os autores consideraram o logaritmo do valor absoluto da diferença entre o Lucro Antes dos Impostos sobre o Lucro, Lucro Tributável e Prejuízo Fiscal não aproveitado no período.

O Lucro Tributável não é amplamente evidenciado pelas companhias que negociam ações nos Estados Unidos, por isso, Hanlon et. al (2012) usou a extrapolação para o cálculo desse lucro. A extrapolação foi realizada pela diferença entre a Despesa de Imposto de Renda e a Despesa de Imposto de Renda Diferido, escalonado pela alíquota máxima de 35\% nos Estados Unidos.

Na presente pesquisa, a extrapolação utilizada tomou como base a alíquota máxima de 34\%, que pode vir a ser paga no contexto brasileiro. Além disso, como não há obrigatoriedade de evidenciação do prejuízo fiscal nas notas explicativas, optou-se, por eliminar essa variável do cálculo como já desenvolvido em outros estudos anteriores como o de Costa e Lopes (2015) e Marques et al. (2016).

No modelo original, Hanlon et. al (2012) utilizam a participação das companhias auditadas no mercado exterior com base no lucro antes dos impostos estrangeiros (FGN no texto dos autores). A divulgação dessa informação nas demonstrações financeiras não é compulsória pela CVM. 
Contudo, conjecturou-se que poderia estar disponível nos complementos das demonstrações contábeis publicadas. A título de verificação por amostragem para validação, foram analisadas algumas companhias por critérios de amostragem aleatório. Observou-se que o lucro antes dos impostos estrangeiros não foi divulgado em nenhum dos casos analisados. Assim, em substituição à variável 'FGN' em Hanlon et al (2012) foi utilizada como proxy se as empresas brasileiras emitem recibos de valores mobiliários em bancos depositários no exterior (ADR). Essa variável é considerada uma proxy para complexidade das operações e assumirá valor igual a 1 nos anos de emissão de ADRs e anos posteriores a ela e 0 para as empresas que não emitiram ADR. Esta classificação é fundamentada no pressuposto de que a emissão de ADRs aumenta a complexidade das operações das empresas auditadas. Assim, a expectativa é de que os honorários de auditoria sejam maiores não somente no ano de emissão de ADR, mas que sejam maiores para as companhias que, em algum momento, foram emissoras destes recibos quando comparadas àquelas que não o foram.

Destaca-se ainda, que Hanlon et al. (2012) utilizaram o logaritmo do número de segmentos operacionais, uma vez que essa é a informação disponível na Compustat. As informações por segmento, nesta pesquisa, foram coletadas diretamente nas notas explicativas das demonstrações financeiras das companhias abertas brasileiras. Diferentemente dos Estados Unidos, em que apenas segmentos operacionais são divulgados de forma segregada, no Brasil, algumas companhias apenas divulgam o que elas chamam de segmentos por região geográfica. Na maioria dos casos, observou-se que não há informações que sejam capazes de elucidar se os segmentos por região geográfica são operacional e conceitualmente semelhantes aos segmentos de negócios mencionados nas IFRS. Por isso, coletou-se o número de segmentos por região geográfica e por segmentos de negócios, e utilizou-se o logaritmo do número de segmentos por região geográfica para aquelas empresas que não divulgaram o número de segmentos operacionais.

Para a estimação do modelo principal, utilizou-se a Equação 1:

$$
\begin{aligned}
& H A_{i t}=\alpha_{i}+\beta_{1} \text { BTD total }_{i t}+\beta_{2} A C C R_{i t}+\beta_{3} B I G 4_{i t}+\beta_{4} A T_{i t}+\beta_{5} S E G_{i t}+\beta_{6} A D R_{i t} \\
& \quad+\beta_{7} E S T_{i t}+\beta_{8} R E C_{i t}+\beta_{9} D I V_{i t}+\beta_{10} R E N_{i t}+\beta_{11} N E G_{i t}+\beta_{12} \text { OPIN }_{i t}+\varepsilon_{i t}
\end{aligned}
$$

Em que:

$H A=$ Honorários de Auditoria, calculado pelo logaritmo dos honorários de auditoria evidenciados no Formulário de Referência da CVM.

$B T D$ total $=$ Diferença total entre o Lucro Contábil (LC) e o Lucro Tributável (LT), calculada conforme Equação 2. Alternativamente foi substituída pela BTD permanente, BTD temporária, BTD positiva, BTD negativa, BTD anormal e BTD normal, conforme respectivas Equações apresentadas adiante.

ACCR $=$ Accruals, calculados pelo logaritmo do valor absoluto da diferença entre o LAIR e o fluxo de caixa líquido das operações.

BIG4 = Status da Firma de Auditoria, em que a dummy é igual a 1 para as empresas auditadas por uma Big4, e 0 caso contrário.

$A T=$ Ativo, estimado logaritmo do ativo total.

$S E G=$ Segmentos, estimados pelo logaritmo do número de segmentos operacionais. No caso das companhias que consideram somente as regiões geográficas como segmentos reportáveis, foi considerado o logaritmo do número de regiões.

$A D R=$ Complexidade das Operações, em que dummy assume valor 1 quando as empresas emitiram ADRs no ano $t$ ou nos períodos anteriores, e 0 caso contrário.

$E S T=$ Estoques, valores dos estoques de curto e longo prazo, escalonado pela média do Ativo.

$R E C=$ Recebíveis, valores das contas a receber de curto e longo prazo escalonadas pela média do ativo total.

$E N D=$ Endividamento, valores do passivo oneroso de curto e longo prazo escalonado pela média do ativo total.

REN = Rentabilidade, estimado pelo Earning Before Interest and Taxes (EBIT) escalonado pela média do ativo total.

$N E G=$ Resultado Negativo, em que a dummy assume valor igual a 1 se o resultado da empresa i for negativo no período t ou nos dois últimos períodos, e 0 caso contrário. 
$O P I N=$ Opinião de Auditoria, em que a dummy assume valor igual a 1 se a empresa i recebeu opinião de auditoria modificada no ano t, e 0 caso contrário.

A BTD total foi estimada conforme especificado na Equação 2.

$$
\mathrm{BTD} \mathrm{total}_{\text {it }}=\log \left(\operatorname{LAIR}_{\mathrm{it}}-\left(\frac{\left(\mathrm{DIRC}_{\mathrm{it}}-\mathrm{DIRD}_{\mathrm{it}}\right)}{\mathrm{A}_{\mathrm{it}}}\right) \mathrm{I}\right.
$$

Em que:

$L A I R=$ Lucro antes do Imposto de Renda da empresa i no ano t.

$D I R C=$ Despesa com Imposto de Renda Corrente da empresa i no ano t.

$D I R D=$ Despesa com Imposto de Renda Diferido da empresa i no ano t.

$A=$ Alíquota máxima dos tributos sobre o lucro da empresa i no ano t.

Estimou-se a BTD permanente e BTD temporária como em Hanlon et. al (2012), demonstradas nas Equações 3 e 4:

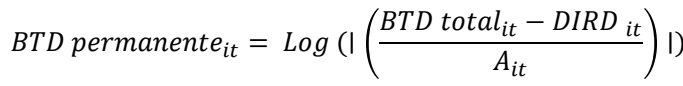

$$
\begin{aligned}
& B T D \text { temporária }{ }_{i t}=\log |D I R C|
\end{aligned}
$$

Onde:

$B T D$ total $=$ Valor estimado mediante aplicação da Equação 2 da empresa i no ano t.

$D I R D=$ Despesa com Imposto de Renda Diferido da empresa i no ano t.

$A=$ Alíquota máxima dos tributos sobre o lucro da empresa i no ano $\mathrm{t}$.

$D I R C=$ Despesa com Imposto de Renda Corrente da empresa i no ano t.

A BTD positiva e a BTD negativa foram identificadas mediante a diferença ano a ano e para cada empresa, entre o LAIR e o LT, como demonstrado nas Equações 5 e 6:

$$
\begin{aligned}
& \text { BTD positiva }_{i t}=\log \left(\left|\operatorname{LAIR}_{i t}-\mathrm{LT}_{i t}\right|\right), \text { se } \mathrm{LC}_{i t}>\mathrm{LT}_{i t} \\
& \text { BTD negativa }_{i t}=\log \left(\left|\mathrm{LAIR}_{i t}-\mathrm{LT}_{i t}\right|\right), \text { se } \mathrm{LC}_{i t}<\mathrm{LT}_{i t}
\end{aligned}
$$

Quando o LAIR foi maior que a extrapolação do LT, a diferença foi denominada BTD positiva. Por outro lado, quando o LAIR foi menor que a extrapolação do LT, denominou-se essa diferença como BTD negativa.

Da aplicação do modelo econométrico utilizado por Tang (2006) e Martinez e Passamani (2014) em estudos anteriores (Equação 7), estimou-se as parcelas normal e anormal da BTD.

$$
\text { BTD total }{ }_{i t}=\alpha_{i}+\beta_{1} \mathrm{INV}_{\mathrm{it}}+\beta_{2} \Delta \mathrm{REV}_{\mathrm{it}}+\beta_{3} \mathrm{NOL}_{\mathrm{it}}+\beta_{4} \operatorname{DIRD}_{\mathrm{it}}+\beta_{5}(\mathrm{PL}-\mathrm{LL})_{\mathrm{it}}+\beta_{6} \Delta \mathrm{EST}_{\mathrm{it}}+\varepsilon_{i t}
$$

Em que:

$B T D$ total $=$ Diferença total entre o LAIR e o $L T$ da empresa i no ano $t$, obtida pela Equação 2.

$I N V=$ Somatório das contas investimentos, imobilizado, intangível e diferido (saldo remanescente da conta, caso exista) da empresa i no ano $t$, ponderado pelo Ativo defasado.

$\triangle \mathrm{REV}=$ Variação da receita da empresa $\mathrm{i}$ do ano t-1 para o ano t, ponderada pelo Ativo defasado. 
$N O L=$ Despesa Corrente com Imposto de Renda quando positiva da empresa i no ano $t$, dividido por 0,34. Caso o IR/CSLL corrente não seja positivo, assume-se o valor 0.

$D I R D=$ Despesa com Imposto de Renda Diferido da empresa i no ano $t$, ponderada pelo Ativo defasado.

$P L-L L=$ Patrimônio Líquido subtraído do Lucro Líquido da empresa i no ano $t$, ponderado pelo Ativo defasado.

$\Delta E s t=$ Variação dos estoques da empresa i do ano t-1 para o ano t, ponderada pelo Ativo defasado.

As variáveis $I N V$ e $\triangle \mathrm{REV}$ são consideradas proxies para mudanças na economia. Assim, quanto maior for o valor para essas variáveis, maior será a probabilidade de existirem despesas indedutíveis, o que culminará na expectativa de sinal negativo para o coeficiente de ambas (Martinez \& Passamani, 2014).

A variável NOL é uma proxy para prejuízo fiscal, tendo em vista que o prejuízo fiscal compensado geralmente não é evidenciado nas demonstrações contábeis. Martinez e Passamani (2014) comentam que essa ausência pode ser explicada pela determinação estabelecida no art. 510 do Regulamento de Imposto de Renda (RIR/1999), que estabelece o máximo de 30\% do lucro líquido ajustado pelas adições e exclusões previstas no respectivo regulamento, como limite para compensação. Quanto ao sinal, espera-se que coeficiente seja positivo, pois à medida que os prejuízos fiscais forem compensados haverá uma variação positiva na BTD (Martinez \& Passamani, 2014).

A variável DIRD é uma proxy para controlar os efeitos dos prejuízos fiscais em virtude de esta variável ser constituída por diferença temporária entre as normas fiscais e societárias. Desse modo, se esta variável for positiva, pode-se dizer que a constituição de diferença temporária foi maior que a compensação de diferença temporária passadas. Quanto ao coeficiente, espera-se que este seja negativo, o que impactaria positivamente na $B T D$.

A variável $P L-L L$ é considerada pelo fato de, no Brasil, os juros sobre o capital próprio poderem ser dedutíveis da base de cálculo dos tributos sobre o lucro, o que impacta significativa na BTD (Martinez \& Passamani, 2014). Em razão de esta variável ser base de cálculo para a distribuição de juros sobre o capital próprio, que é dedutível para fins fiscais, estima-se que seu coeficiente seja positivo.

A variável $\triangle \mathrm{EST}$ é pertinente pelo fato de os estoques das companhias possuírem "em seus valores de formação parcelas relacionadas a incentivos ligados à produção (custos) e alterarem a formação de BTD conforme a realização ou não desses estoques" (Piqueiras, 2010, p. 55).

Considera-se que os resíduos da Equação 7 sejam a BTD anormal, pois referem-se a parcela da BTD que não pode ser explicada pelas variações nas receitas e no prejuízo fiscal, nível de investimentos em ativos tangíveis e intangíveis. A parcela da $B T D$ que pode ser explicada por essas variáveis é considerada como diferença normal (BTD normal), e foi obtida pela diferença entre a BTD total e a BTD anormal.

Para os testes de sensibilidade a fórmula de cálculo da BTD especificada na Equação 2 foi substituída por aquelas aplicadas por Costa e Lopes (2015) para o contexto brasileiro na determinação do LT (Equação 8), BTD total (Equação 9) BTD temporária (Equação 10) e BTD permanente (Equação 11).

$$
\text { Lucro Tributável }{ }_{i t}=\frac{\text { DIRC }_{\text {it }}}{A_{i t}}
$$

Em que:

$D I R C=$ Despesa com Imposto de Renda Corrente da empresa i no ano $\mathrm{t}$

$A=$ Alíquota máxima dos tributos sobre o lucro da empresa i no ano $\mathrm{t}$.

$$
\text { BTD total }{ }_{\text {it }}=\frac{\left(\mathrm{LAIR}_{\text {it }}-\mathrm{LT}_{\text {it }}\right)}{\mathrm{AT}_{\mathrm{it}-1}}
$$

Onde:

$L A I R=$ Lucro antes do Imposto de Renda da empresa i no ano t.

$A T=$ Ativo total defasado da empresa i no período i no período t-1. 


$$
\text { BTD temporária }{ }_{i t}=\frac{\left(\mathrm{DIRD}_{\mathrm{it}} / \mathrm{A}_{\mathrm{t}}\right)}{\mathrm{AT}_{\mathrm{it}-1}}
$$

Em que:

$D I R C=$ Despesa com Imposto de Renda Corrente da empresa i no ano $\mathrm{t}$

$A=$ Alíquota máxima dos tributos sobre o lucro da empresa i no ano $\mathrm{t}$.

$A T=$ Ativo total defasado da empresa i no período i no período $\mathrm{t}-1$.

$$
\text { BTD permanente }_{i t}=\text { BTD total }_{i t}-\text { BTD temporária }_{i t}
$$

Sendo:

BTD permanente $=$ Diferença permanente entre o lucro societário e o lucro fiscal da empresa i no ano t.

$B D T$ total $=$ Diferença total entre o lucro societário e o lucro fiscal da empresa i no ano t.

BTD temporária $=$ Diferença temporária entre o lucro societário e o lucro fiscal da empresa i no ano t.

A Equação 7 foi novamente utilizada para estimação da parcela normal e anormal da $B T D$, mas desta vez considerou-se como variável resposta os valores de $B T D$ encontrados da aplicação da Equação 9. A magnitude da $B T D$ dos testes de sensibilidade também foi analisada, atribuindo-se as denominações BTD positiva ou BTD negativa, conforme descrito nas Equações 5 e 6.

\section{REFERÊNCIAS DO MATERIAL SUPLEMENTAR}

Agoglia, C. P., Doupnik, T. S., \& Tsakumis, G. T. (2011). Principles-based versus rules-based accounting standards: The influence of standard precision and audit committee strength on financial reporting decisions. The Accounting Review, 86(3), 747-767.

Costa, P. S., \& Lopes, A. B. (2015). Implicações da adoção das IFRS sobre as book-tax differences: o caso do Brasil. Alemanha: NEA.

Fávero, L. P., Belfiore, P. P., Silva, F. L., \& Chan, B. L (2009). Análise de dados: modelagem multivariada para tomada de decisões. Rio de Janeiro: Elsevier.

Hanlon, M., Krishnan, G. V.,\& Mills, L. F. (2012). Audit Fees and Book-Tax Differences. The Accounting Review, 34(1), 55-86.

Marques, A. V. C., Costa, P. S., \& Silva, P. R. (2016). Relevância do Conteúdo Informacional das Book-Tax Differences para Previsão de Resultados Futuros: Evidências de Países-Membros da América Latina. Revista Contabilidade \& Finanças, 27(70), 29-42.

Martinez, A. L., \&Passamani, R. R. (2014). Book-Tax Differences e sua Relevância Informacional No Mercado de Capitais No Brasil. Revista de Gestão, Finanças e Contabilidade, 4(2), 20-37.

Piqueras, T. M. (2010). Relação das diferenças entre o lucro contábil e o lucro tributável (book-tax differences) e gerenciamento de resultados no Brasil. Dissertação de mestrado, Faculdade de Economia, Administração e Contabilidade, Universidade de São Paulo, São Paulo.

Tang, T. Y. H. (2006). The Value Relevance of Book-Tax Differences - An Empirical Study in China's Capital Market. SSRN Eletronic Journal. 